\title{
A portable, solid-state anomaloscope
}

\author{
ROBERT T. KINTZ \\ Eastman Kodak Company, Rochester, New York
}

\begin{abstract}
Various methods of assessing color vision are discussed. The anomaloscope is concluded to be the best of these, but it is not widely used because of complexity and cost considerations. The design of a portable, solid-state anomaloscope that uses tristate light-emitting diodes is described. This device has many of the desirable features of the standard Nagel-type anomaloscope and can be constructed at a fraction of the price.
\end{abstract}

Many devices and techniques, each with its own particular advantages and deficiencies, have been devised to assess color vision. The pseudoisochromatic plates that are most commonly used are composed typically of a field of printed dots or patches that vary in hue and saturation. Persons with normal color vision are able to perceive some figure when they view these plates, whereas persons with a color defect cannot. In another type of pseudoisochromatic-plate test, persons with a color-vision defect perceive one pattern, and color normals see another. Such devices are very portable and relatively inexpensive. Unfortunately, they require a very specific viewing illuminant in order for the test to be valid, a factor that is often neglected in practice. Also, the power of such instruments to discriminate among the various types of color deficiency and their extents is limited.

Another variety of color-vision test requires an observer to arrange a number of small, colored patches into a series along some hue axis. Usually, there are several of these hue series to be arranged in order to cover a significant portion of color space, so that an observer often requires 10 to $15 \mathrm{~min}$ to complete the test. Here again, the spectral composition of the viewing illuminant is a critical factor determining test validity. Although, in terms of diagnostic ability, tests of this type are generally more powerful than the pseudoisochromatic plates, they do not have the potential for definitive diagnosis that is required for scientific investigation.

A superior device for such purposes is the anomaloscope, which Boynton (1979) dubbed "the undisputed queen of all color-vision testing instruments." The principle on which the anomaloscope is based is fundamental and simple-namely, that a spectral "yellow" color can be matched by some mixture of two other spectral primaries, "red" and "green." The red/green ratio in the mixture required for the match is often called the "Rayleigh quotient," after Lord Rayleigh,

The author's mailing address is: Health, Safety \& Human Factors Laboratory, Eastman Kodak Company, Rochester, New York 14650. who discovered that this single quantity is distinctive for color normals and can be used to distinguish among normals, anomalous trichromats, and dichromats.

Although many designs based upon Rayleigh's discovery (see review by Willis \& Farnsworth, Note 1) have been proposed for anomaloscopes, the most popular commercial implementation of the principle is the Nagel anomaloscope. With the Nagel system, an observer views a bipartite field of from 1 to $3 \mathrm{deg}$ of visual angle, as shown in Figure 1. Half of this field, usually the bottom, is illuminated with a monochromatic yellow $(589 \mathrm{~nm})$ that can be varied in intensity with a knob. The other half of the field is composed of the red/green mixture $(670$ and $545 \mathrm{~nm})$, the ratio of which can be varied by a second control knob. The observer is required to adjust the two knobs until an exact match between the two halves of the field has been achieved. The test is simple, quick, and direct. Usually, several settings are required for reliability, but the testing time is very short compared with the methods discussed above, and the spectral quality of ambient illumination is not a critical factor.

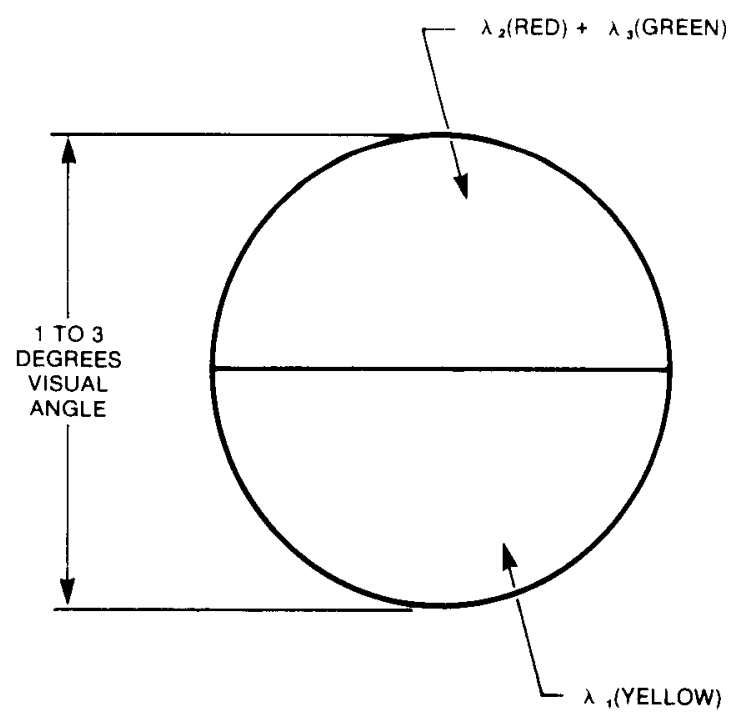

Figure 1. Field of view typically found in Nagel anomaloscope. 
Major disadvantages of the anomaloscope, disadvantages that have precluded its wide adoption as a clinical test instrument, are its complexity and cost. The generation and precise control of monochromatic stimuli typically entail highly regulated power supplies, expensive optical filters, lenses, and wedges, and welldesigned mechanical linkages for varying both the red/ green ratio and the luminance. The instrument is consequently large, heavy, delicate, and expensive. Portable versions have been designed and built (Piantanida, 1976), but they are usually scaled-down versions of laboratory instruments and suffer from the same complexity/cost considerations. The anomaloscope, despite its superior capability, is found normally only in laboratories concerned with serious color-vision research.

\section{A SOLID-STATE DESIGN}

With solid-state microelectronics, an anomaloscope that is both small and lightweight, as well as inexpensive, can be designed. Moreover, since it has no moving parts, it is substantially less fragile and less subject to misalignment or calibration errors. The device on which such a design is based is the light-emitting diode, or LED (see review by Nygaard \& Frumkes, 1982). LEDs emit narrow-bandwidth illumination, comparable to that used in filter anomaloscopes, at various points in the visible spectrum as a function of their chemical composition. LEDs with peak outputs close to those used as primaries in conventional anomaloscopes are now available, and their output is stable and their power consumption low. Saunders (1976) built an anomaloscope using LEDs, but his design employed discrete devices and required Maxwellian view optics to produce sufficiently useful light output.

Particularly advantageous in the design of a truly portable, electronic anomaloscope is the tristate LED, so named because it has two "on" states (differing in spectral output) as well as the "off" state. In reality, the tristate LED is actually two (or more) LEDs mounted in a single package, in which the two (or more) LEDs are wired in parallel, but in opposite polarity (shunt opposition). This has the convenient consequence that, when current of positive polarity is applied to the device, one of the LEDs is "on" and the other "off"; a negative polarity turns the second LED "on" and the first "off." When the polarity of the input is varied rapidly to achieve a frequency higher than can be detected by the human visual mechanism, the tristate LED is perceived to be continuously "on" and to have a color that is a mixture of the individual LED output wavelengths. Finally, if the duty cycle (negative/positive polarity proportion) of the suprathreshold flicker frequency is made to vary, any mixture of the two LED outputs can be generated. By choosing a tristate LED with red and green output primaries, any mixture, or Rayleigh ratio, of light outputs between the two primaries can be achieved.
A new series of tristate LEDs, recently announced by Hewlett-Packard, has proved to be satisfactory for application in this design approach. The Hewlett.Packard applications literature refers to these as "LED bicolor light bars." One type, the HP-2950, is a high-efficiency red/yellow LED combination in an 8-pin DIP package. It contains four individual tristate LEDs in a single package approximately $1 \mathrm{~cm}$ square. The yellow LEDs in this package have a dominant wavelength of $585 \mathrm{~nm}$, which is very close to the yellow primary of the Nagel anomaloscope. These are used for the standard yellow field in the present design. The red-LED half of the HP-2950 is left unused.

The red/green field is produced with a second HewlettPackard bicolor light bar LED in the same package configuration, the HP-2965. The HP-2965 has four LEDs with peak wavelength outputs at $635 \mathrm{~nm}$ (red) and $565 \mathrm{~nm}$ (green).

Circuitry to drive the two LEDs is shown in Figure 2. IC2 is used to control the luminous intensity of HP.2950, the yellow primary in one half of the anomaloscope field. IC2 is an LM555 tirner circuit configured to permit independent control of the output duty-cycle polarity at a constant output frequency. As R5 is varied, one period is decreased and the other is increased proportionately. In the configuration shown, the duty cycle can range from about $0.01 \%$ to $99.99 \%$ with very little change in frequency.

ICI is another LM555 timer used exactly as is IC2, but in this case the change in duty cycle is used to control the relative "on"/"off" proportions of the red and green primaries of HP-2965. IC3 is an LM556 dual timer used, in this instance, as an inverter with pushpull outputs. Pin 2 (THR) is used as an enable control, and is driven by the output of IC1. When THR is high, the red LED is "on"; when THR input is low, the green LED is "on." Pin 4 (RST) is shown tied high; however, an additional 555 circuit such as IC1 or IC 2 can be used as input to RST to permit the experimenter a calibration/ intensity control of HP-2965.

All component values shown are for $\mathrm{Vcc}$ equal to $15 \mathrm{~V}$. R5 and R2 are the observer's control knobs for the adjustment of the yellow intensity and red/green ratio, respectively. Diode D5 and the associated 500-ohm potentiometer serve to regulate the current flow to each LED and equalize their luminous outputs. This control is necessary because of the greater barrier potential of the green LED and makes it possible to ensure the same forward current's flowing through each diode. Capacitors $\mathrm{C} 1$ and $\mathrm{C} 2$ have been chosen for frequencies of approximately $1000 \mathrm{~Hz}$, which is well above the frequency range at which flicker of even the highest modulation can be perceived.

\section{CALIBRATION AND MECHANICAL DETAILS}

Several approaches are available for the readout of observer settings of potentiometers R2 and R5. In the 


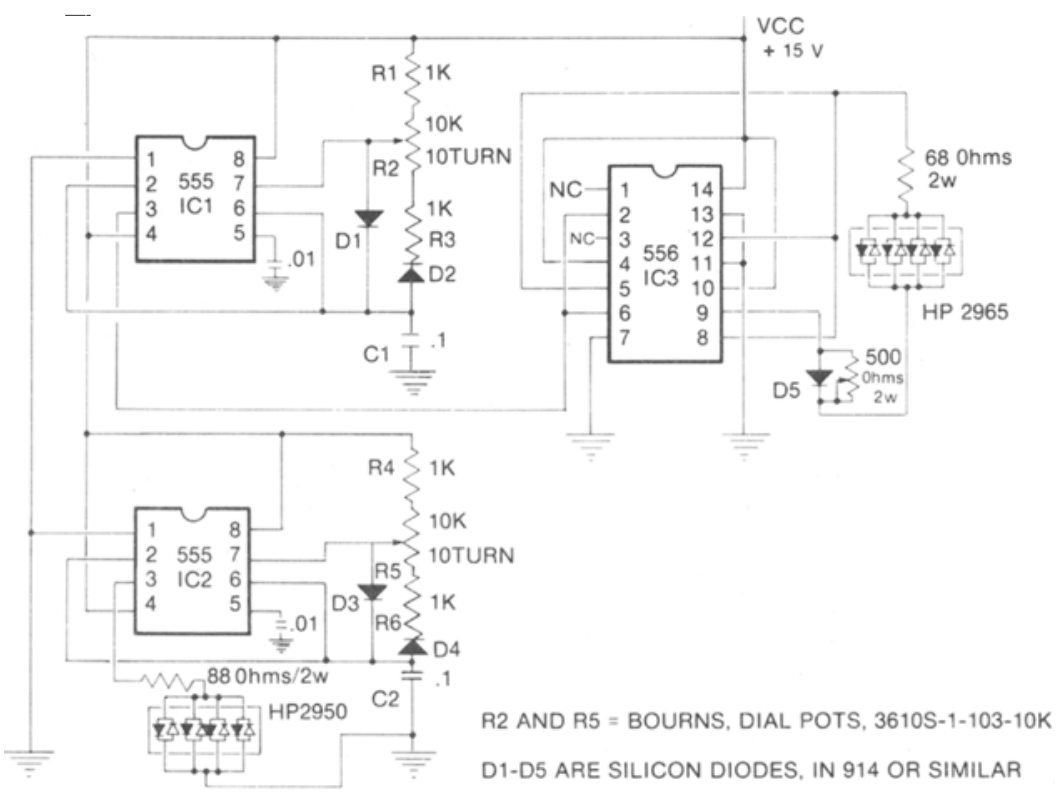

Figure 2. Schematic diagram of a solid-state anomaloscope.

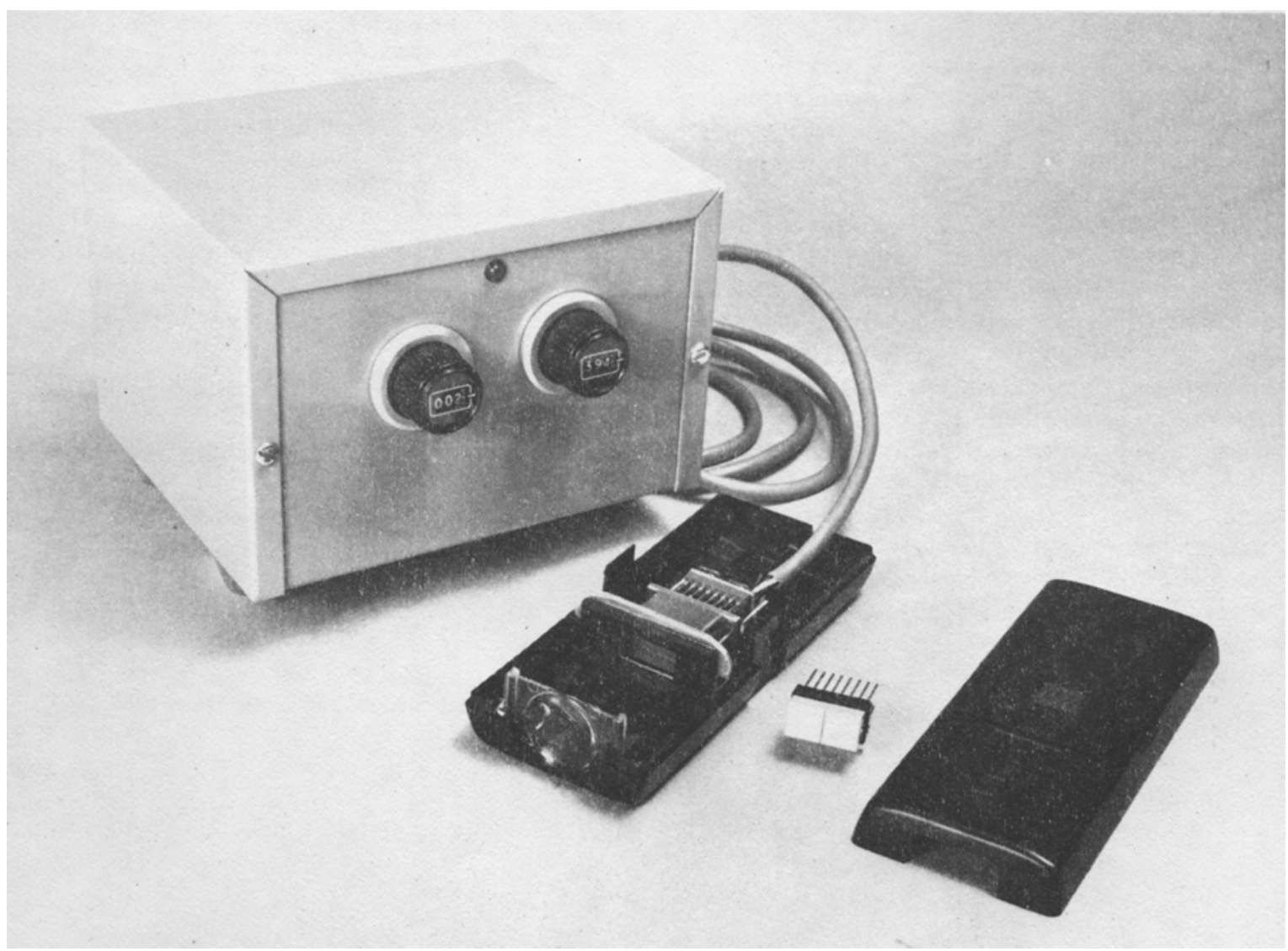

Figure 3. Solid-state anomaloscope, with hand-held viewer disassembled to show the mounting of the LEDs, mirror-box asembly, and flashed opal glass viewing screen. 


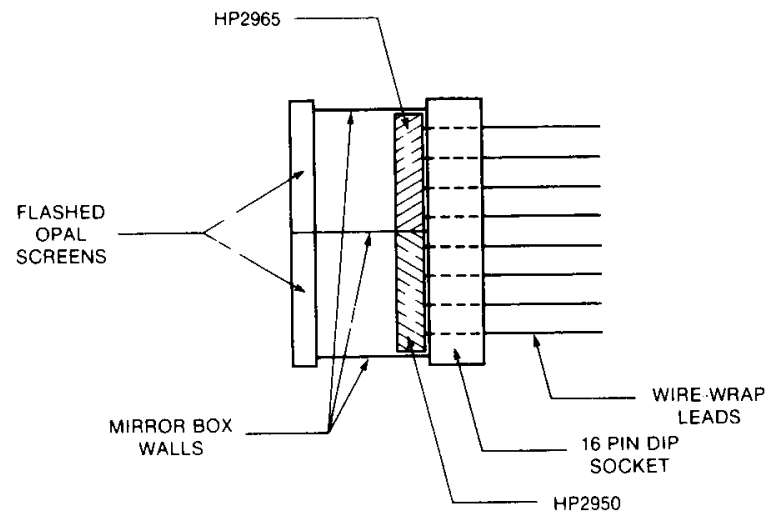

Figure 4. Cross-section through mirror-box and LED lightbat assembly to show details of the design and construction.

laboratory, the duty-cycle changes produced by these settings can be monitored on an oscilloscope, and potentiometer scales calibrated for field use. The use of multitum potentiometers having integral digital tumscounters of up to four digits will make the readings of observers' settings easy and less prone to error. (We have used pots by Bourns, part No. 3610S-1-103-10K; see Figure 3.) Additional optical components required for this device are both minimal and conventional. Both of these LED light bars produce fields of moderately high luminance in the circuit described (approximately $100 \mathrm{~cd} / \mathrm{m}^{2}$ at match conditions produced by a colornormal observer), but field uniformity is less than entirely satisfactory for anomaloscope application directly. To overcome this deficiency, the LED DIP packages were mounted side by side in a 16-pin DIP socket. Next, a small, internally mirrored light box (Cavonius, 1974) was constructed around each, with a flashed opai glass viewing surface located approximately .25 -in. from the light-bar surface, as shown in Figure 4. This arrangement allows sufficient internal reflections from the mirror-box walls and in the flashed opal glass screen to produce uniform fields of high brightness. Alternatively, an integrating bar of clear acrylic, such as that described by White, Wolbarsht, and Tieger (1975), can be used. The mirror box or integrating bar, and the LED DIP package, can be mounted in a hand-held enclosure such as a $35-\mathrm{mm}$ slide viewer, as shown in Figure 3, with a cable attaching it to the remote electronics and power supply. The entire package can be constructed for less than $\$ 150$.

The hand-held viewer shown in Figure 3 produces a bipartite field of approximately $3.5 \mathrm{deg}$ subtense. We have recently constructed another version of the instrument that is a desk-top, free-viewing model. The moderately high luminance of the instrument permits this configuration in subdued lighting conditions. Caution must be observed, however, to make sure that no direct light falls on the field viewing surface. Also, the observer should be positioned such that the field subtends between 1 and 2 deg. Pokorny, Smith, Verriest, and Pinckers (1979) noted that specific problems with dichromats and anomalous trichromats arise when the field is outside this range.

Before the device can be used to assess color-vision anomalies, the range of settings by normal observers must be established. Until a standardized version of the instrument becomes available, slight differences in component values or construction practice dictate that norms for each anomaloscope be established individually after construction.

\section{REFERENCE NOTE}

1. Willis, M. P., \& Farnsworth, D. Comparative evaluation of anomaloscopes (Research Report No. 190; Project No. NM 003 041.26.01). New London, Conn: Bureau of Medicine and Surgery, Department of the Navy, August 1952.

\section{REFERENCES}

Boynton, R. M. Human color vision. New York: Holt, Rinehart \& Winston, 1979.

Cavonius, C. R. A method for mixing lights to produce homogeneous illumination. Behavior Research Methods \& Instrumentation, 1974, 6, 29-30.

NygaARd, R. W., \& Frumkes, T. E. LEDs: Convenient, inexpensive sources for visual experimentation. Vision Research, $1982,22,435-440$.

Piantanida, T. P. A portable filter anomaloscope. Optical Engineering, 1976, 15, 325-327.

Pokorny, J., Smith, V. C., Verniest, G., \& Pinckers, A. (Eds.). Congenital and acquired color vision defects. New York: Grune \& Stratton, 1979.

SAunders, J. E. A red-green anomaloscope using light emitting diodes. Vision Research, 1976, 16, 871-874.

White, C. W., Wolbarsht, M. L., \& Tieger, T. A fast visual colorimeter. Behavior Research Methods \& Instrumentation, 1975, 7, 260-264.

(Manuscript received August 25, 1983; revision accepted for publication December $9,1983$. 